\title{
TV/Series
}

$6 \mid 2014$

Écho et reprise dans les séries télévisées (III) : de la métafiction à la transmédialité

\section{À l'intérieur de Seinfeld : la sitcom Jerry}

\section{Eric Gatefin}

\section{(2) OpenEdition}

\section{Journals}

Édition électronique

URL : http://journals.openedition.org/tvseries/321

DOI : 10.4000/tvseries.321

ISSN : 2266-0909

Éditeur

GRIC - Groupe de recherche Identités et Cultures

Référence électronique

Eric Gatefin, «À l'intérieur de Seinfeld : la sitcom Jerry », TV/Series [En ligne], 6 | 2014, mis en ligne le 01 décembre 2014, consulté le 02 mai 2019. URL : http://journals.openedition.org/tvseries/321 ; DOI :

$10.4000 /$ tvseries.321

\section{(c) (i) (9)}

TV/Series est mis à disposition selon les termes de la licence Creative Commons Attribution - Pas d'Utilisation Commerciale - Pas de Modification 4.0 International. 


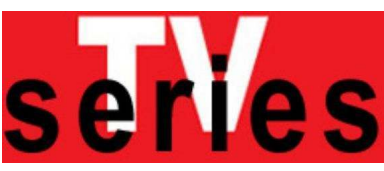

À l'intérieur de Seinfeld : la sitcom Jerry

Éric GATEFIN

L'arc narratif principal de la saison 4 de Seinfeld, diffusée sur NBC, consiste en une mise en abyme de la création du show. Cette saison s’intègre en fait dans un processus transgressif global. L'écriture de Seinfeld repose en effet sur une dénonciation sans concession des conventions sociales, des dogmes moraux mais aussi des codes télévisuels liés aux sitcoms. Cette dénonciation s'opère sur le mode de la charge. Montrer ce qui échappe en principe au regard, tel semble être le projet des scénaristes qui, dans leur entreprise de démystification des tabous, incluent les mécanismes de production de la sitcom Seinfeld.

Les étapes de création du show présentées dans la saison 4 font l'objet d'un traitement corrosif. De l'absence fondamentale d'idées de la part des personnages soi-disant créatifs aux pitches douteux, en passant par une négociation financière peu reluisante, la série avance cahin-caha vers sa création. Au-delà des soubresauts qui conduisent de la rencontre avec NBC à la diffusion d'un épisode, le caractère provocant de la mise en abyme vient principalement du concept arboré fièrement par le personnage de George : « a show about nothing ", concept qui met au premier plan la démarche critique et démystificatrice de la série.

Loin de figurer comme une astuce scénaristique, l'arc narratif relatant la fabrication du show exhibe au contraire la volonté de déconstruction à l'œuvre tout au long de la série. Il entre également dans un jeu d'échos au sein d'une sitcom qui fait de l'auto-référence son mode de fonctionnement principal. Ainsi, les étapes qui conduisent à la réalisation du pilote s'articulent aux questions sexuelles traitées dans plusieurs épisodes: la virginité, la masturbation, la frustration, l'homosexualité et l'impuissance. De manière directe ou implicite, la création et les pulsions sexuelles des personnages interfèrent et s'éclairent entre elles.

$\mathrm{U}$

n comique new-yorkais, ses amis, ses amours. Ainsi résumée, la sitcom Seinfeld (NBC, 1989-1998) ne semble pas devoir surprendre le téléspectateur. Produite peu avant des séries comme Dream on (HBO, 1990-1996), Friends (NBC, 1994-2004) ou Mad About You (NBC, 1992-1999), elle entretient avec elles de fortes parentés thématiques. Situations, personnages, gags, rythme, le téléspectateur ne peut qu'être parfois troublé par ces univers si voisins. Il est aussi nécessairement dérouté par la singularité de Seinfeld, à l'intérieur de ce champ en apparence si balisé. L'arc narratif principal de la saison 4 est à cet égard emblématique de l'audace des auteurs de Seinfeld : voilà le personnage principal Jerry Seinfeld, qui semble jouer son propre rôle, contacté pour créer une sitcom sur le network NBC. Tout est donc en place pour une stricte mise en abyme de la série, avec ses risques et ses complexités.

Comment ce processus est-il montré dans la sitcom? Considérée sur l'ensemble de la saison, la représentation de la fabrication d'une série semble reprendre assez fidèlement les étapes principales de production : contacté par NBC, le personnage de Jerry 
Seinfeld saisit l'opportunité de voir une série bâtie autour de lui. Avec le concours de son ami George, il propose un concept. Après un accord de principe ont lieu les négociations sur le salaire. Une fois soumis le scénario d'un pilote, les auteurs attendent le verdict des décideurs de la chaîne. La décision leur est favorable. George et Jerry sont donc invités à choisir des comédiens lors d'un casting, avant que le tournage n'ait lieu dans le dernier épisode de la saison.

Derrière ce résumé linéaire se cache une grande disparité de traitement des différentes étapes. D'abord, cet arc narratif, qui donne bien une perspective d'ensemble à la saison, n'est pas évoqué dans quelques épisodes tandis qu'il est présent seulement en filigrane dans d'autres. Ensuite, ce qui intéresse paradoxalement les auteurs de la série, c'est la mise en scène de l'écriture - la recherche du concept d'abord, de l'histoire ensuite et des dialogues. Le casting et le tournage n'occupent que l'épisode final de la saison, alors qu'ils semblent plus aisés à montrer. Cette prégnance s'explique par le dispositif de la sitcom. Contrairement à certains autres formats télévisés, ce genre est par excellence celui de l'écriture. L'auteur, au sens où la critique française l'entend, ne peut être que celui qui imagine dialogues, situations, entrelacs des lignes d'intrigue, puisque le metteur en scène suit une grammaire visuelle particulièrement basique. Ainsi, nous montrerons que Larry David et son équipe proposent moins à voir une mise en abyme directe de la série au sens où un épisode serait montré dans un épisode, qu'une image de leur travail d'écriture de la série. La mise en branle de la sitcom Jerry offre un nouveau moyen de mettre à jour le mécanisme de la création et d'exhiber le fonctionnement de cette machine à effets que constitue tout programme télévisé.

\section{La petite histoire d'une création}

Seinfeld porte sur le processus même de la réalisation d'une série le même regard acide et décapant que sur les autres domaines qu'il aborde au cours de chaque épisode. On trouve dans cette saison 4 la trace d'un certain nombre de critiques habituelles contre le médium télévisé et ses productions. Reste à voir jusqu'où les créateurs peuvent aller, d'une part compte tenu de la censure qui s'exerce sur leurs scripts, d'autre part pour ne pas heurter un public qui verrait d'un mauvais œil une critique trop radicale de ce qu'il est en train de regarder.

\section{Satire de l'univers télévisé}

La satire de l'univers télévisé est étrangement poussée assez loin tout au long de la saison. Pour une série diffusée sur un network 
généraliste (NBC), le fait peut surprendre. Plusieurs éléments viennent expliquer cette relative liberté accordée aux auteurs de la sitcom. D'abord, Seinfeld n'est pas jusque-là une série phare de la chaîne. Diffusée après la très populaire Cheers (NBC, 1982-1993), elle retient l'attention d'un public de fidèles mais ne connaîtra un large succès que lors de cette quatrième saison ${ }^{1}$. Par ailleurs, dans leurs premiers développements, les péripéties de la création de la série paraissent ridiculiser principalement les auteurs de sitcoms. Seule une vue rétrospective sur l'ensemble de la saison permet de saisir le caractère corrosif du propos pour l'ensemble de ce milieu.

De prime abord, les dirigeants de NBC passent simplement pour assez naïfs et peu regardants sur la valeur des programmes qui leur sont proposés. Leur goût paraît assez peu sûr, à l'inverse de leur sens de la négociation financière qui tournera en défaveur des deux auteurs. Une vue plus détaillée sur le destin du projet conduit par les personnages de Jerry et George montre que les incertitudes qui entourent la création de ce pilote sont d'abord entretenues par les fluctuations des désirs et caprices des dirigeants. C'est parce que Susan, conseillère du président de la chaîne, va désirer George que les auteurs auront une chance d'écrire leur pilote ; c'est parce que George va jeter un œil un peu trop insistant sur le décolleté de la fille du producteur de NBC que la réalisation de ce même pilote sera remise en cause. Enfin, si le producteur change d'avis, c'est qu'il est obsédé par Elaine, après avoir été charmé, à son tour, par son décolleté. Rejetées au second plan, les interrogations sur la valeur de la création proposée ne jouent guère de rôle dans la mise au point du pilote. Ce dernier est subordonné à un chantage sexuel, dont créateurs et producteurs sont parfaitement conscients et dont ils savent jouer quand l'occasion se présente.

Du côté du public, la même indifférence au contenu règne. Si les producteurs de NBC n'ont guère à se préoccuper des programmes qu'ils diffusent, c'est aussi parce que les téléspectateurs n'ont pas la moindre exigence artistique sur ce qu'ils regardent. À Jerry qui s'inquiète de ne pouvoir écrire pour la télévision, George rétorque : "Come on Jerry, it's just TV » (4.3). Meuble central de l'appartement de Jerry, la télévision est accaparée par les personnages et fonctionne comme une drogue, qui conditionne les individus. Regards habités, fascinés s'abîment dans la contemplation de jeux ou de programmes enfantins ${ }^{2}$. L'invisible enfant-bulle auquel George et

${ }^{1}$ Sur ce point, comme sur les conditions de production de la saison 4 de Seinfeld, voir le documentaire «La saison du succès » dans les bonus du coffret de la saison 4, chez Sony Pictures (2005).

${ }^{2}$ Dans l'épisode «The Contest » (4.11), Jerry escompte maîtriser ses pulsions sexuelles en regardant un programme pour enfants. 
Susan rendent visite est un tyran domestique par la maîtrise qu'il a de la télécommande (4.7). Son caractère acariâtre passe aisément pour la conséquence de son immersion permanente dans l'univers du petit écran. Kramer, incapable de maîtriser son addiction, fait fi de ce qui l'entoure pour mieux participer à la version américaine de Jéopardy (4.10), comme dans cette scène :

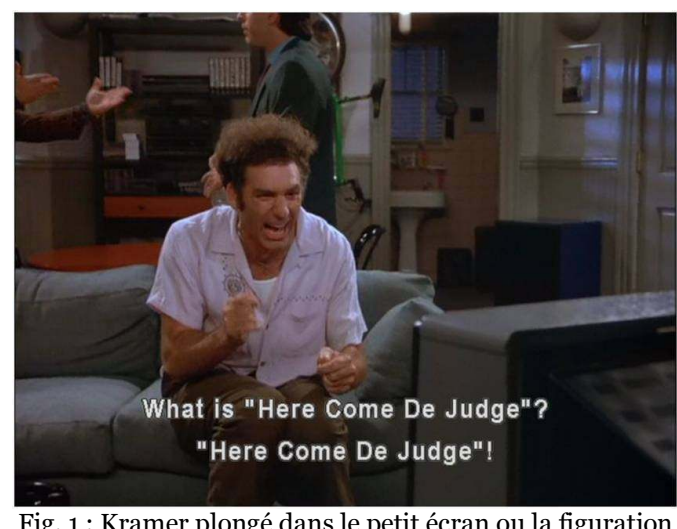

Fig. 1 : Kramer plongé dans le petit écran ou la figuration de l'absence complète de distance avec la télévision

L'intégration de ses répliques dans le flux du dialogue entre Jerry et George souligne l'absence totale de relation à l'autre induite par le rapport fusionnel au téléviseur.

La sitcom Jerry créée par George et Jerry n'a pas vocation à dépareiller dans cet univers où superficialité et abrutissement sont de mise. Réaffirmé à plusieurs reprises, le projet des deux auteurs est d'être simplement amusant. Jerry ambitionne de donner une nouvelle ampleur à sa carrière de comique. George pense pouvoir gagner beaucoup d'argent et séduire des femmes grâce à ce métier. Bien vite, cependant, les auteurs de Seinfeld donnent à voir la réalité face à ces fantasmes. Maigrement rétribué pour l'écriture du pilote, George est par ailleurs raillé par une femme qui souligne la médiocrité du genre de la sitcom.

On voit que la critique de l'univers de la télévision est acerbe. Conformément au ton général de la série, le rire se veut grinçant, dans la mesure où sont visées toutes les instances participant à la création d'une sitcom : la charge contre les producteurs devient notamment, par degrés, particulièrement sévère tout comme celle qui vise le public. La satire de la création audiovisuelle atteint toutefois une toute autre portée, quant on touche au contenu du programme proposé et à 
l'écriture de la sitcom. Cette fois, les auteurs de la série Seinfeld se prennent en quelque sorte pour objet de la création et offrent une étrange représentation de leur activité.

\section{«A show about nothing »}

Il est assez significatif que l'initiateur du projet de série soit George. Conçu après quelques tâtonnements, son concept se veut, au choix, révolutionnaire ou absurde. Il prétend imaginer « a show about nothing » $(4.3$, voir figure 2$)$ :

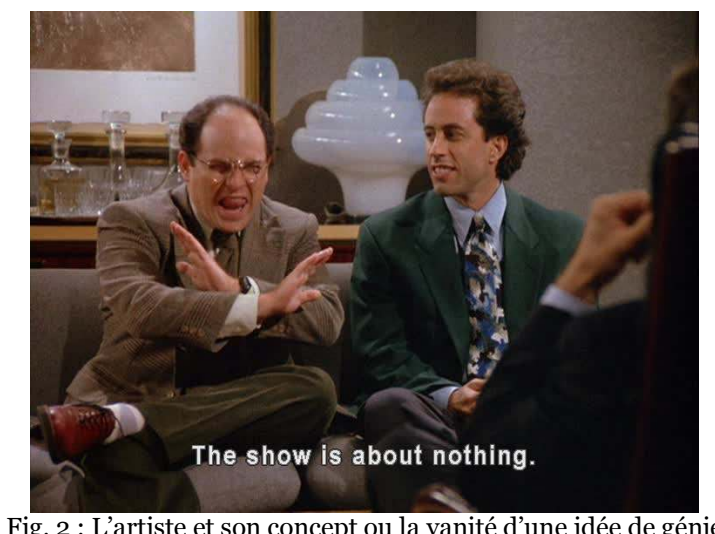

Encore convient-il de définir ce "rien », sujet de débat avec les producteurs de NBC mais aussi entre George et Jerry. Dans une première acception, le « rien » signifie l'absence de récit progressif dirigé vers une fin et contenant un message. En somme, la fiction ne dirige pas l'esprit du spectateur vers un univers imaginaire qu'il s'agirait de représenter et donc d'abord de concevoir. Se substitue à ce récit classique une peinture de la vie quotidienne, de ce qu'on pourrait appeler des riens. Il s'agit de petits détails a priori sans intérêt, sans importance, qui prennent pourtant une place inopportune dans l'esprit de chacun et qui se trouvent à leur place à la télévision, écran destiné au familier plus qu'au spectaculaire.

Mais ce projet, George semble vouloir le radicaliser, passant des petits riens au Rien avec un grand « $\mathrm{R}$ » : il n'est plus alors question de mettre en relief certains aspects de la vie quotidienne mais de la représenter dans son absolue banalité, voire en en ôtant tout ce qui pourrait être de l'ordre de l'événement. À la question de 
Dalrymple: «Why am I watching it?», George, triomphal, réplique : «Because it's on TV! » (voir figure 3 ).

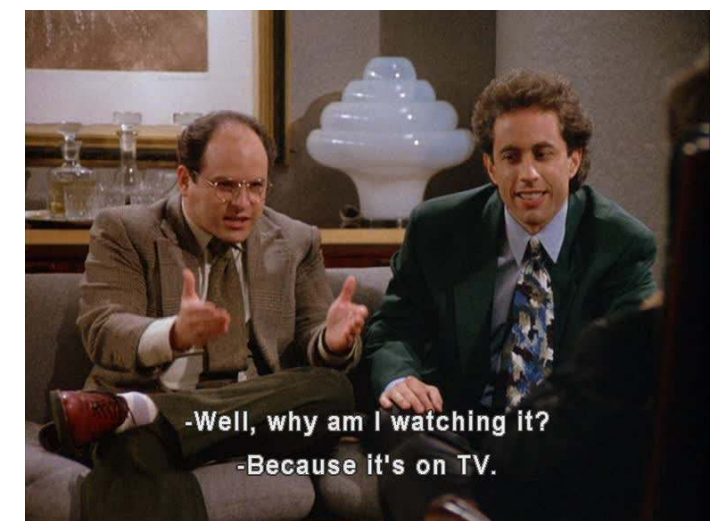

Fig. 3 : Vers la téléréalité : le programme comme flux sans contenu

En somme, le média occupe une place tellement banale dans la vie de tous les jours que le programme conçu n'a plus d'importance en soi. On n'est pas loin du projet de la téléréalité, du moins sur le principe. Cette idée s'accorde particulièrement à la paresse de George dans la mesure où l'écriture consiste alors à collecter des faits, sans invention ni travail de dramatisation.

Or, très vite, pour George comme pour les concepteurs de programmes bas de gamme, la question du contenu se pose à nouveau et implique un changement de perspective. La concession du créateur ne règle pourtant rien. Après le vide revendiqué vient le vide subi, signe d'impuissance. Longtemps, Jerry reste une coquille vide que les deux auteurs ne savent pas remplir. Des pitches hasardeux qui ne mènent nulle part, une absence de motivation dans les idées qui feraient plus qu'agacer un Robert Mc Kee, des dialogues navrants ne dépassant pas « Hello ! » et « How are you doing ? », Jerry semble bien appelée à rester un rien (4.8). Cette sitcom est le signe de la paresse de ses auteurs à consentir le moindre effort intellectuel et à développer une idée personnelle. Dès lors, le temps pendant lequel les deux auteurs devraient composer Jerry est consacré à trouver un prétexte pour ne pas écrire, à dormir ou encore à regarder la télévision. Processus d'évitements, mais aussi d'aveuglement, car dans le même temps que le scénario ne se construit pas, Jerry et George vivent une existence scénarisée avec brio, dont Jerry repère même, pour le téléspectateur, le caractère brillamment construit (4.10). Il fait ainsi remarquer à George 
que sa situation présente un aspect singulier. D'abord, il a obtenu une situation socialement enviable en séduisant Susan : le voilà auteur de sitcom. Mais l'obtention de ce statut ne lui permet pas d'en bénéficier pour séduire une femme puisqu'il ne le détient que dans la mesure où il est le petit ami de Susan ${ }^{3}$. Coïncidence et ironie du sort, voilà deux artifices d'écriture que les auteurs de Seinfeld pointent aux yeux du téléspectateur par l'intermédiaire du dialogue, comme si la mise à jour du procédé ne nuisait pas à son efficacité comique. Surtout, cette mise en exergue est à double détente, puisqu'une autre ironie du sort échappe aux personnages. En effet, leurs situations vécues fourniraient la matière de la fiction au second degré qu'ils désespèrent d'inventer.

Passant ainsi à côté de ce qui constituerait le contenu d'une sitcom dans leur existence, George et Jerry lancent un pitch typique d'un comique de situation, sans intérêt aux yeux de Jerry, mais qui retient toute l'attention des producteurs. D'un concept audacieux on passe donc à une histoire conventionnelle peu vraisemblable. Jerry ne sera pas « a show about nothing ».

\section{Une fiction-miroir : Jerry dans Seinfeld}

L'objet final obtenu constitue-t-il une mise en abyme de Seinfeld, au sens d'une représentation fidèle de la fiction au premier degré ? Si Jerry n'est pas une sitcom sur rien, peut-elle être perçue comme un double exact de Seinfeld?

Le trouble principal introduit par la mise en scène du tournage du pilote consiste dans l'identité des acteurs-personnages. Un écart se crée entre les acteurs, lié à celui qui transparaît dans la sitcom Seinfeld. À un premier niveau en effet, Jerry Seinfeld interprète son propre rôle dans la série que nous regardons. Son personnage a donc un statut particulier par rapport à ceux d'Elaine, George et Kramer. Effet redoublé ici : Seinfeld joue son rôle en train de jouer son rôle dans la sitcom Jerry. Problème : cette mise en abyme ne fonctionne pas de la même manière pour ses comparses. George, Elaine et Kramer, personnages de fiction, sont repris dans la fiction au second niveau sous les traits d'acteurs jouant le rôle d'acteurs censés les interpréter (voir figure 4).

3 Pour un autre exemple, voir l'épisode « The Cheever letters » (4.8) : George a reçu du père de Susan des cigares cubains qu'il n'aime pas. Il les a offerts à Kramer. Plus tard, invité à passer un week-end dans la cabane que le père de Susan possède, il a vu cette habitation partir en fumée à cause de la négligence de Kramer qui a déposé un cigare dans cette cabane. George aura bien du mal à faire apprécier au père de Susan toute l'ironie de la situation. 


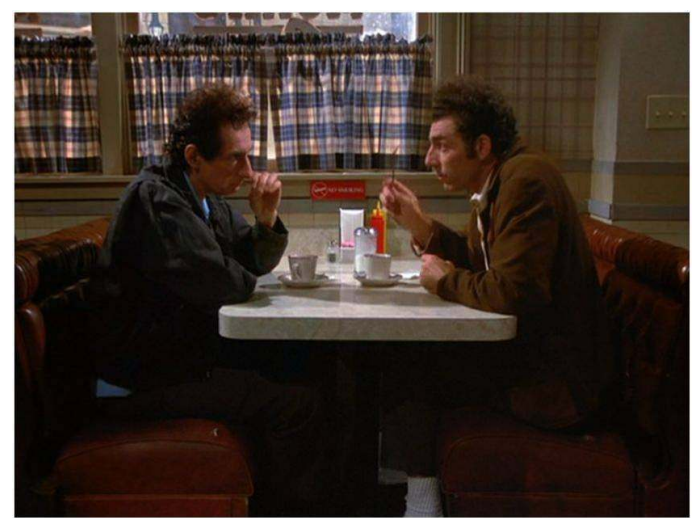

Fig. 4 : Kramer et son double : Michael Richards face à l'acteur qui aurait pu jouer son rôle dans la fiction Seinfeld

Les acteurs interprétant les personnages de la fiction au second degré induisent tous un trouble dans leur rapport avec leur rôle (4.23 et 24). Le Kramer au second degré se détache du Kramer joué par Michael Richards qui convoitait le rôle. Il est indépendant, marginal et retrouve paradoxalement les traits du personnage de Kramer en refusant la fusion, l'imitation. L'actrice qui interprète Elaine veut au contraire s'immerger totalement dans son personnage. Puisqu'Elaine a couché avec Jerry, elle le fera aussi. Elle rompra aussi dans une scène où, pour elle, fiction et réalité semblent se mêler. Enfin, le comédien interprétant George panique tant à l'approche du tournage qu'il entre ainsi dans le rôle même qu'il craint de ne pouvoir jouer. Le spectateur de Seinfeld se retrouve également face à un trouble compte tenu des ressemblances physiques entre les personnages et les acteurs les incarnant dans la fiction au second degré. Tout se passe comme si ces acteurs étaient des caricatures des personnages de Seinfeld, ayant avec eux une vague parenté mais paraissant simultanément incapables d'incarner à nos yeux Elaine, George et Kramer.

Au-delà de la question des acteurs se pose celle du contenu de la série. Le téléspectateur n'a qu'une perception fragmentaire de ce qu'est le pilote Jerry. Les auditions, le tournage offrent quelques éléments, qui semblent indiquer que les personnages recyclent des répliques et situations de leur vécu dans la fiction Seinfeld. Pour autant, ces échos ne s'articulent pas à l'histoire du pilote. Ils sont autant de morceaux détachés, de références sans lien avec l'intrigue appelée à être traitée. De manière significative, les trois scènes montrées lors de la diffusion du pilote sont entrecoupées des réactions de différents personnages de la saison devant leur petit écran. Ainsi, le caractère fragmentaire de ce 
qui est montré est souligné. Dans le même temps, les effets comiques sont plus efficaces dans les scènes de téléspectateurs, car ils fonctionnent en écho avec des situations issues des autres épisodes de la saison, ce qui est impossible pour le pilote de Jerry. Plus exactement, la seule façon dont les extraits de Jerry peuvent faire rire tient aux liens qu'ils entretiennent avec la fiction Seinfeld. Le rire est alors pure affaire de ressemblance, de familiarité mais il est simultanément désamorcé par le décalage qui fait que Jerry n'est pas un épisode de la fiction Seinfeld mais bien une autre fiction peu innovante en tant que telle.

Incapable d'adhérer au jeu d'acteurs interprétant des personnages au second degré, à un pitch peu enthousiasmant et à une sitcom qui semble singer la fiction au premier degré sans en capter la singularité, le téléspectateur ne peut, à l'instar de la nouvelle productrice, que porter un jugement négatif sur le produit final. Difficile en réalité de définir clairement les intentions des auteurs de Seinfeld dans les extraits du pilote diffusé. Il semble qu'il leur fallait nécessairement attester de l'existence de la fiction au second degré en en proposant des images, mais il est évident qu'ils avaient plus à dire sur le processus de création que sur son aboutissement. Ce qui ressort justement de ce processus est l'idée d'une vanité absolue, qui tient autant aux créateurs qu'aux récepteurs du projet. De cette vanité naît Jerry, une sitcom comme les autres, mais ce qui transparaît en filigrane, c'est bien sûr que ce vide absolu est aussi celui de Seinfeld, de sorte qu'à travers la conception de la sitcom, c'est bien à une vertigineuse mise en crise de la fiction que le lecteur assiste (voir figure 5).

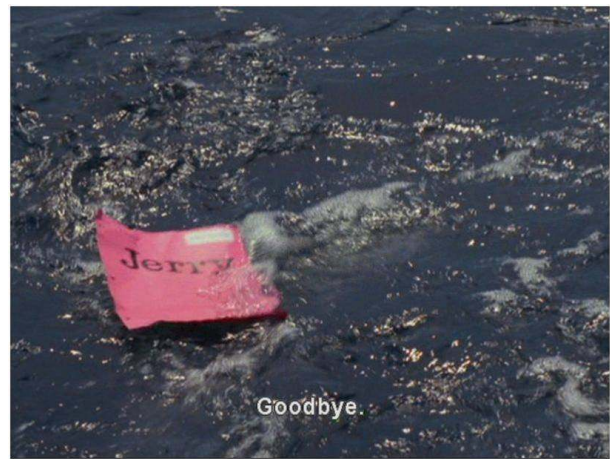

Fig. 5 : Du naufrage d'un texte à celui d'une fiction 


\section{George, « the nothing man »}

\section{George, personnage principal}

Cette crise de la fiction est avant tout, me semble-t-il, celle d'un personnage qui fait exploser le cadre classique de la sitcom. George est, en effet, de manière assez paradoxale, le personnage central de la série Seinfeld. On sait que l'écriture de la sitcom est supervisée, pendant les sept premières saisons, par Larry David et Jerry Seinfeld. Le premier a volontiers reconnu avoir beaucoup mis de lui dans la figure de George, avant d'en venir à une autoreprésentation directe dans Curb your Enthousiasm 4 (HBO, 2000-2011). Le concept initial et les apparences mettent en exergue Jerry Seinfeld. Donnant son titre à la série et interprétant son propre rôle, il accueille le téléspectateur et les autres personnages dans son appartement. Des extraits de ses spectacles de stand-up ponctuent chaque épisode pendant les sept premières saisons. Les personnages d'Elaine, Kramer et George sont censés graviter autour de lui, au point que dans un épisode, George et Elaine éprouvent de la gêne à rester tous les deux (3.4), Seinfeld étant, finalement, leur seul point commun. Figure indispensable, Jerry vaut donc d'abord comme lien. Aux yeux du téléspectateur, il peine parfois à exister pleinement comme personnage. À l'inverse, tout en déroutant complètement les habitudes du téléspectateur, George apparaît d'emblée comme une figure nettement caractérisée. Son instabilité, sa singularité lui valent d'imprimer son ton et son rythme à la série.

\section{Un danger pour la fiction}

Co-créateur de la série Jerry, George est à la fois l'élément moteur et l'obstacle fondamental de la production du programme. Face à un Jerry d'abord inhibé, il estime qu'il est facile d'écrire pour la télévision, vu ce qui y passe. Il est ensuite à l'initiative de pitches peu brillants mais qui témoignent de son implication. C'est au final l'une de ses idées peu pertinentes qui servira de base à la réalisation du pilote. Par ailleurs, George est l'inventeur du concept du «show about nothing ", concept-limite qui lui vaut la méfiance des dirigeants de NBC. Sa relation instable avec Susan, le regard qu'il dirige vers le décolleté de la fille du président de NBC (4.17) sont autant d'erreurs qui remettent en cause l'existence de la série. Symboliquement donc,

${ }^{4}$ La question du récit spéculaire est au cœur des créations de Larry David. Il poussera plus avant encore le travail de mise en abyme de Seinfeld dans Curb your Enthousiasm qui fait intervenir les acteurs de la première série dans leur identité de comédiens traçant leur route après l'arrêt de Seinfeld. Il n'est évidemment pas anodin que Larry David ait aussi fini par interpréter un rôle principal chez Woody Allen (Whatever Works, 2009), cinéaste lui auss préoccupé par la question de l'auto-représentation dans plusieurs de ses films, parm lesquels on peut citer Crimes and Misdemeanors (1989) ou Deconstructing Harry (1997). 
Larry David et Jerry Seinfeld, auteurs de la série, font de George celui qui est au fondement et qui empêche simultanément la fiction de se faire. La mise en abyme offre une lecture de la fonction du personnage de George dans la sitcom Seinfeld : il est à la fois la condition sine qua non de l'existence de la série (quel intérêt aurait-elle sans lui ?) et ce qui fait obstacle à son aboutissement, en ce sens peut-être qu'il l'empêche d'être une sitcom comme les autres et de se dérouler suivant des codes classiques. Les pathologies du personnage, son caractère souvent antipathique, perpétuellement dysfonctionnel enraient les processus classiques d'attachement et de continuité du récit. George, en effet, se montre incapable d'apprendre de ses erreurs; il ne manifeste aucun désir de s'amender et condamne son entourage à suivre le même processus. Ainsi, la figure de Jerry Seinfeld, peu marquée au début de la série, rejoindra progressivement celle de George. Initialement chargé de l'analyser, de mettre à jour ses nombreuses pathologies, Jerry incarne d'abord le discours de la norme. Mais, progressivement, son personnage tend, avec son caractère propre, à évoluer suivant une mécanique identique à celle de George 5 . Spectateur de ses errances, il en devient parfois aussi l'initiateur en même temps qu'il expose lui aussi au grand jour ses tares et multiples obsessions $^{6}$. Ainsi, il apparaît que, dans une grande mesure, George entraîne les autres et l'ensemble de la sitcom Seinfeld, vers le vide et la folie.

\section{Portrait d'un instable}

À l'instar des grandes figures de marginaux, George est un être des extrêmes. Quand il défend l'idée d'une "série sur rien ", il est, selon les points de vue, génial ou ridicule. Mais son attitude même à l'égard de cette idée passe d'un extrême à l'autre. D'un sentiment de supériorité, il passe à une compromission totale en renonçant à ce à quoi il semblait croire. Dans les autres domaines, George navigue ainsi d'une position à l'autre. Après avoir maudit Susan, employée de NBC à laquelle il doit tout mais qui l'empêche de séduire d'autres femmes (4.10), il se mue en amoureux désespéré, déclamant sa passion sous le regard indifférent de ses amis auxquels il se donne en spectacle (4.13). Plus que de sentiments sujets à variation, c'est bien le rôle qui importe et surtout la compassion et l'intérêt qu'ils peuvent provoquer. Tout se

${ }^{5}$ Sur ce point, voir aussi Nils Ahl et Benjamin Fau (dir.), Dictionnaire des séries télévisées, Paris, Philippe Rey, 2011, p. 792.

${ }^{6}$ Le premier épisode de la septième saison ( "The Engagement ») offre un bon exemple de cette évolution. George et Jerry prennent la résolution de donner un sens à leur existence en faisant preuve d'une plus grande maturité dans leurs relations sentimentales respectives, ceci devant les conduire au mariage. Tandis que George franchit le pas avec Susan, Jerry hésite et rompt le pacte, témoignant d'une inaptitude viscérale à évoluer. 
passe comme si George se trouvait face à un auditoire et qu'il continuait à interpréter son rôle malgré la perplexité de son public, concentré sur une fiction en laquelle il est le seul à croire. À peine Susan reconquise, il cherchera d'ailleurs immédiatement comment rompre avec elle (4.13). Ce grand écart est aussi celui de la hiérarchie entre l'essentiel et l'accessoire. Au moment où la série qu'il a contribué à concevoir s'apprête à voir le jour (4.24), George est miné par deux préoccupations : il veut savoir s'il est atteint d'un cancer et si l'acteur interprétant Kramer a réellement volé des raisins secs lors de son audition. Ce qui est ainsi mis en scène, c'est la manière dont l'esprit du personnage oblitère ce qui pouvait être un enjeu majeur pour lui. Il substitue à cela deux obsessions d’importance tout à fait inégales. Ce jeu des disproportions sur lequel les auteurs construisent les lignes d'intrigue concernant George permet à de multiples reprises de remettre en cause les conventions sociales et les normes de comportement. Fréquemment, l'attitude de George lève certains tabous : il est prêt à rompre avec sa compagne qui vient d'être renvoyée de son emploi par sa faute (4.10) et négocie une réduction pour un voyage par avion en profitant de la mort de la tante de sa petite amie (4.19). On voit que si George est un personnage des extrêmes, il n'est jamais question pour les auteurs de lui attribuer la moindre forme de grandeur.

Ces contradictions et cette faiblesse morale font de George un être vain. Pour rompre avec une compagne trop éprise, il affiche sa vacuité, proclamant: "I'm nothing » (4.16), écho évident de l'expression « a show about nothing » (voir figure 6).

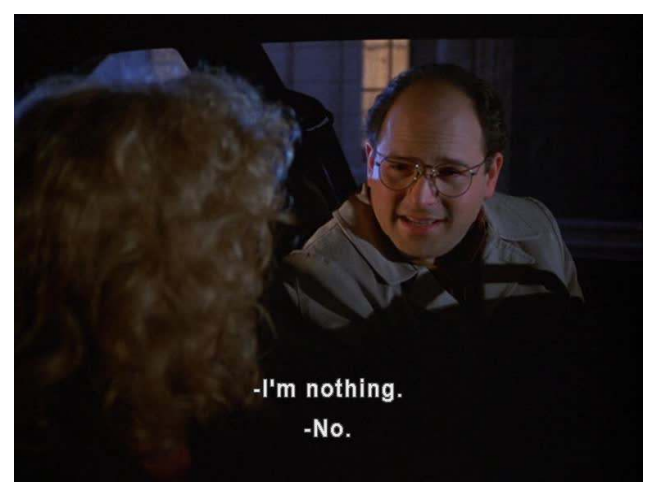

Fig. 6 : Un personnage à l'image de sa fiction :

la vacuité assumée 
Mais même de cette insignifiance, George ne parvient pas à convaincre son interlocutrice. Il lui faudra par conséquent endosser des rôles pour espérer susciter le dégoût de sa compagne. Il sera d'abord un homosexuel, ensuite une star de cinéma pornographique, sans que ces changements d'identité lui permettent de démontrer son insignifiance à la jeune femme. La déviance entraîne inévitablement la déviance, et tout semble indiquer que cette compagne est elle-même dérangée. Ce spectre de la folie rôde significativement autour de George. Parmi les personnages récurrents de cette quatrième saison se trouve Joey De Vola, qui fréquente le même psychiatre qu'Elaine. Sa première apparition coïncide avec un moment de panique chez George avant de rencontrer les dirigeants de NBC. Une continuité apparaît alors entre les deux personnages, tous deux aspirants scénaristes. Tandis que De Vola menace la vie de Jerry, George présente un sérieux danger pour sa réussite professionnelle. La suite confirmera ce risque : Jerry conseille à George d'aller voir une assemblée de psychiatres pour traiter son cas après sa conduite désastreuse lors du rendez-vous à NBC. Aussi incontrôlable que l'inquiétant De Vola, George verbalise ce que l'humanité ordinaire enfouit, il agit souvent en dépit du bon sens et se meut dans son propre univers, guidé par ses obsessions.

Que nous dit finalement George sur les figures de la série? En bien des occasions, elles ne font qu'endosser des rôles sociaux sans y croire véritablement. Une sorte de distance s'établit entre le personnage et le rôle qu'il devrait tenir. Par ailleurs, c'est l'ensemble des conduites déviantes qui trouvent un lieu de manifestation dans la série. La norme guette, la censure morale, la réprobation aussi mais de manière pulsionnelle, viscérale émerge l'anormalité triomphante, fruit d'un fonctionnement social absurde par ses conventions. Quel récit construire alors? Comment redonner sens à ce qui n'en a pas ?

\section{Variations critiques}

Larry David et Jerry Seinfeld ne vont pas mettre leurs ressources d'écriture au service du récit. S'affranchissant des codes en se donnant pour visée la maxime « No hugging, no learning »7, ils fondent un nouveau registre d'attachement ou, plus exactement, de séduction. On sait qu'une sitcom repose sur la répétition, que la familiarité joue un rôle essentiel. De ce point de vue, Seinfeld suit les codes en mettant en place un univers étriqué, réduit à quelques

\footnotetext{
${ }^{7}$ Que l'on pourrait traduire par « Pas de câlins, pas de transmission de savoir ».
} 
décors ${ }^{8}$. Mais de manière assez retorse, les auteurs font aussi un usage dévoyé de la répétition, source d'une démarche critique de commentaire. Les rapprochements les plus osés et improbables sont établis pour nourrir un comique qui ne naît plus alors de la scène proprement dite et des traits d'humour qu'elle contient mais de la relation qui se met en place entre des éléments disparates. Série de la reprise et de la variation, Seinfeld nourrit son rire d'une prise de distance et d'un dispositif qui ravale au même rang des éléments auxquels on n'assignerait pas a priori la même valeur.

\section{Combinaisons virtuoses}

L'épisode «The implant» (4.19) peut donner une idée de ce système : les situations, les dialogues entrent dans un jeu de références vertigineux, déployé tout au long de l'épisode, mais qui va aussi fonctionner à l'échelle de la saison et de l'ensemble de la série. Prenons à un premier niveau une situation-clef: tout comme Jerry se demande s'il a à faire à une femme qui s'est fait refaire les seins, Kramer voudrait savoir si c'est bien Salman Rushdie qui fréquente le club de gym. On voit que l'écart est maximal entre une situation frivole et une autre qui implique une personnalité mêlée à une actualité grave ${ }^{9}$. Ce doute est à l'origine d'une enquête, de renversements de situation qui s'articulent autour d'une scène-clef qui établit la connexion :

SEINFELD. There is Sidra.

KRAMER. There is Salman.

SEINFELD. Where?

KRAMER. Talking to that woman.

SEINFELD. Talking to Sidra?

KRAMER. If that's Sidra, she's talking to Salman.

SEINFELD. I don't think that's Salman.

KRAMER. Well, I don't think they're real.

SEINFELD. If that's Rushdie, they're real.

KRAMER. If they're real, that's Rushdie.

SEINFELD. Well, I've got to know, I'm talking to Sidra.

KRAMER. I've got to know, I'm talking to Salman. (4.19)

La vitesse du dialogue, les répétitions, parallélismes et chiasmes étourdissent le téléspectateur. Si l'écriture d'une sitcom se nourrit d'échos, on atteint dans Seinfeld certaines limites : d'une part, il y a quelque chose d'irrévérencieux à évoquer les opérations de chirurgie esthétique dans le milieu du spectacle; d'autre part, évoquer la

${ }^{8}$ Seinfeld est, en effet, une sitcom qui fait paradoxalement exploser les cadres d'un genre, sans s'affranchir pour autant du carcan des règles qui le constituent. Sur ce point, voir Vincent Colonna, L'Art des séries télé, Paris, Payot, 2010, p. 276.

9 En l'occurrence, une condamnation à mort pour avoir écrit Les Versets sataniques, roman qualifié de blasphématoire par l'ayatollah Khomeiny en 1989. 
situation de Salman Rushdie s'entraînant à la salle de gym pour pouvoir mieux fuir les Musulmans fondamentalistes constitue une approche plutôt grinçante du drame vécu par l'écrivain. Enfin, la mise en rapport des deux situations constitue une satire d'un principe fondateur de la fiction, qui est la recherche de la vérité. Les deux quêtes présentent un aspect dérisoire et ne font que souligner les traits majeurs des deux êtres assoiffés de vérité : Kramer s'empêtre dans son rapport au réel tandis que Jerry fait montre d'une grande superficialité.

La répétition avec variation, la mise en parallèle n'ont pas vocation à la gratuité, elles sont chargées d'une dimension critique. Le téléspectateur acquiert une distance qui le fait rire en jugeant le personnage en même temps qu'il peut s'amuser de la répétition pour elle-même. L'attitude de George lorsque sa compagne apprend la mort de sa tante donne à voir cette double détente du rire (4.19). Si le téléspectateur s'amuse de voir George mimer en quelque sorte les paroles de la jeune femme, il rit aussi bien sûr de l'inconvenance de son comportement. La caricature qu'offre le mime produit un décalage très caractéristique de la série. La scène est par ailleurs insérée par le montage dans une séquence où Elaine et Jerry discutent de la poitrine de la compagne de celui-ci. Miné de tous côtés, le coup de téléphone tragique devient une nouvelle dérisoire. Le tabou du deuil explose : le personnage incarnant une réaction normale est falot, n'entraîne aucune identification. Seuls importent le dédoublement du discours en mime et le potentiel comique qu'annonce le détachement de George à l'égard de cette mort. À l'échelle de la série, les reprises avec variation de cet épisode abondent. Le personnage de la petite amie fait écho aux relations sentimentales de George avec des personnages fragiles, leitmotiv de la série. Par ailleurs, la dérision appliquée au domaine de la mort fait penser bien sûr à la manière dont George réagit à la mort accidentelle de Susan, qu'il s'apprêtait à épouser par incapacité à rompre (8.1). Ainsi, chaque épisode de Seinfeld devient un puzzle constitué de pièces familières qui produisent des combinaisons surprenantes et entrent en réseaux avec les situations d'autres épisodes.

\section{Les images du bas corporel}

L'arc narratif de la saison 4 se trouve lui-même pris dans ce tourbillon de signes, où les mises en réseaux exploitent sans limite ce qui est de l'ordre du bas corporel. Corps et création sont intimement liés sans que jamais ce lien soit ouvertement énoncé. La première évocation du projet d'une série intervient juste après une discussion où George s'interroge sur l'histoire du papier-toilette à travers les siècles (4.3). Papier fonctionnel produit en grande quantité, le papier-toilette peut être l'équivalent d'une page de sitcom aisément produite, du 
moins selon George. Plus tard, le vomissement viendra se substituer à un échange sur le script rédigé par les deux auteurs de la sitcom Jerry. Pris de nausées, le président de NBC ne sera pas en mesure de formuler ce qui ne convient pas dans le scénario (4.17). On voit bien alors une volonté de mettre de côté l'intellect et la dimension artistique au profit d'un élément qui a trait au corps et à son contrôle. La dévalorisation de la création connaîtra son apogée lors du dernier épisode de la saison, où le tournage du pilote est mis en parallèle avec les problèmes de constipation de Kramer (4.24). Le règlement de cette difficulté intervient en effet juste au moment où la série est enfin diffusée ou, pourrait-on dire, évacuée.

Si la dimension scatologique est régulièrement reprise, ce sont plus encore les métaphores sexuelles qui jouent un grand rôle dans cette saison qui marque une progression dans la liberté de ton de la série par rapport à ces questions. Il semble que, dans une certaine mesure, la mise en abyme de la série et les mises en scène de la virginité, de l'homosexualité et de la masturbation entrent dans une même logique de test des limites de ce que les producteurs et téléspectateurs sont susceptibles d'accepter. L'écriture et les modalités d'approche de ces thèmes constituent le nœud de la série : tout se passe comme s'il s'agissait de montrer au public les risques pris pour ensuite les désamorcer par un traitement habile qui emporte l'adhésion et suscite le rire.

A priori, les épisodes traitant explicitement des questions sexuelles ne sont pas ceux où se déploie l'arc narratif de la création de la série. Pourtant, le jeu des mises en réseaux permanentes dont nous avons vu des exemples fonctionne une nouvelle fois dans ce cas. Le travail d'écriture à deux de Jerry et George offre d'abord une piste pour confirmer leur éventuelle homosexualité. Dans l'épisode "The Outing » (4.16), les deux personnages s'entretiennent avec une journaliste dans une scène aux quiproquos multiples où leur travail en commun passe pour un indice de plus d'une complicité qui dépasse le stade de l'amitié ${ }^{10}$. Plus tôt dans la saison, l'épisode traitant de la virginité (4.10) permet de remettre au premier plan le manque d'expérience des scénaristes ignorant leur métier, maladroits dans l'approche de leur sujet, incapables de se mettre en action. L'épisode « The Virgin » commence ainsi par une scène où Jerry s'inquiète de ne pas être parvenu à écrire - les hésitations de la vierge pour le passage à l'acte feront écho aux excuses que se sont trouvés George et Jerry pour ne pas écrire.

${ }^{10}$ Pour une analyse de cet épisode, cf. Charlotte Gould, «'Not That's There Anything Wrong With That': A Queer Reading of Seinfeld", in GRAAT, issue \#2, June 2007 , http://www.graat.fr/queertvo4.pdf, lien consulté le 10 décembre 2014. 


\section{La masturbation: impuissance ou création?}

Enfin, de manière plus fondamentale, la masturbation traitée dans l'épisode « The Contest » (4.11) s'associe étroitement au processus créatif. Reprenons au moment où George fait preuve d'une absence de fierté risible face aux producteurs de NBC qui lui demandent de revenir sur l'idée d'une série ne parlant de rien. Prêt à tout pour obtenir les faveurs des producteurs, il s'exclame : «Without a story, this is just masturbation!», phrase accueillie avec surprise et réserve par tout l'entourage (voir figure 7).

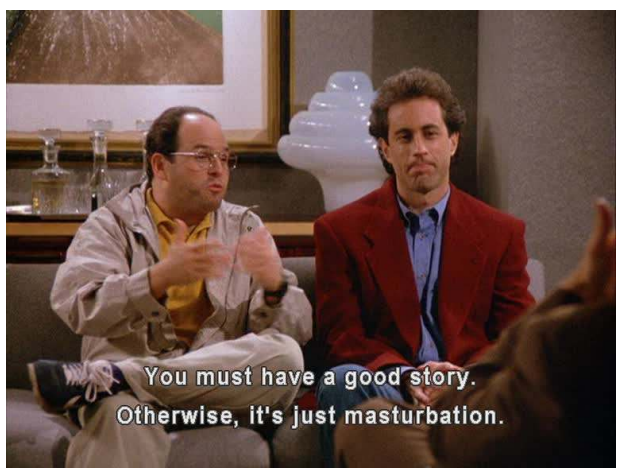

Fig. 7 : Le nouveau dogme de George :

ne pas écrire pour se faire plaisir

En quoi la création d'une série comme Jerry peut-elle être associée à l'onanisme? Une première réponse consisterait à revenir sur la première réaction de Kramer et de George à l'évocation d'une sitcom sur Jerry (4.3). Chacun des deux va proposer des idées incongrues et peu convaincantes. Ces propositions ne tiennent aucun compte de ce qui est susceptible de séduire un large public. Elles reflètent plutôt le caractère déviant ou dérangé des deux personnages qui cherchent à se faire plaisir plutôt qu'à s'interroger sur la validité de leurs idées. La proposition est l'équivalent d'une pulsion irréfléchie où la satisfaction personnelle passe au premier plan. Ainsi, la création ressemble bien dans ce cas à la masturbation : elle passe par un fantasme, une idée personnelle à partir de laquelle l'imaginaire de l'individu travaille. Dans les deux cas, tout destinataire est nié, notamment parce que Kramer et George s'attachent à la première idée qui leur vient à l'esprit.

Autre caractéristique de l'écriture de la sitcom Jerry : composée par deux hommes, elle intègre avec difficulté l'élément féminin. Dans "The Shoes» (4.17), George et Jerry sont prêts à évacuer le personnage d'Elaine, qu'ils ont oublié dans leur pilote et pour laquelle 
ils ne parviennent pas à imaginer le moindre dialogue. Comment savoir ce qui se passe dans l'esprit d'une femme? L'affaire paraît insurmontable. Si Elaine parvient à intégrer la série, ce n'est que par l'intermédiaire de deux scènes de voyeurisme, où la convoitise masculine finit par rendre indispensable une présence féminine. Ce regard concupiscent porté sur la femme sera évidemment l'un des leitmotivs de l'épisode "The Contest» (4.10): George observe une patiente qui se dénude derrière un drap à l'hôpital ; Kramer et Jerry profitent de la présence d'une voisine qui se déshabille face à leurs fenêtres.

Par un savant jeu de miroirs, c'est sur la série Seinfeld que le principe de George finit par trouver un écho. " The Contest », comme épisode, représente au premier degré le sens de la phrase prononcée par George. Puisqu'il n'y a pas d'histoire, il n'est question que de masturbation. C'est pourtant de ce vide que naît un épisode. Ce qui apparaît n'être rien, pur plaisir personnel qui n'a que soi pour visée, se révèle en fait plus productif qu'on ne l'imagine. Comme le dit Jerry un peu plus tôt, « Nothing is something. » (voir figure 8)

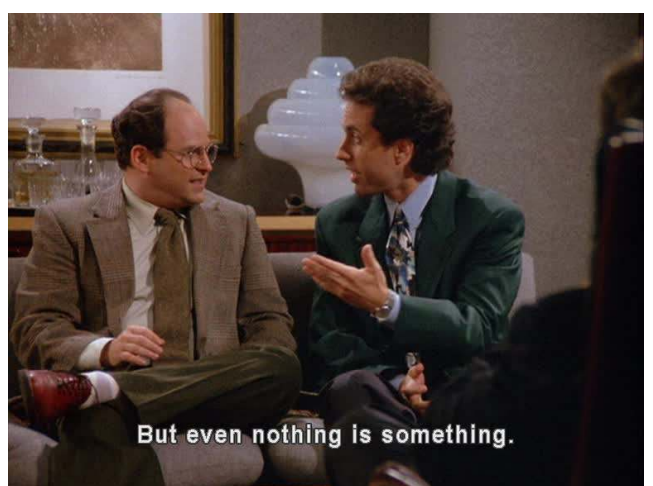

Fig. 8 : Jerry et le compromis théorique : produire à partir du néant

Plus largement, donc, ce qui n'intéresse que soi, qui ne semble avoir de signification que pour soi vaut en réalité d'être partagé. Les histoires anodines vécues par les auteurs ou racontées à eux sont susceptibles d'être investies par autrui. La masturbation associée à l'écriture de la série Seinfeld signifie certes à un premier niveau que les auteurs n'ont rien à transmettre, en termes de valeurs, ou de messages. Constamment réaffirmée, l'idée de transgression présente la particularité de ne produire dans un second temps aucun retour à la norme, à laquelle une morale pourrait aisément se greffer. Mais le triomphe de ce cynisme, de cette méchanceté gratuite demeure une 
création, une production de l'esprit qui, aussi dévalorisée soit-elle, persiste à affirmer son existence. Ainsi, l'impuissance fondamentale à construire un récit classique aux repères identifiables contraste avec le pouvoir des auteurs de bâtir sur des ruines. L'onanisme n'est pas qu'un pis-aller, pour poursuivre la métaphore sexuelle. Il ne fait pas que s'associer négativement aux refoulements et frustrations vécus par les personnages. Ce pur témoignage de l'égotisme est aussi partie prenante de la création. Si mettre en abyme la fiction risque à coup sûr de passer pour un exercice d'autosatisfaction des auteurs s'occupant seulement d'eux-mêmes, c'est encore, quoiqu'il en soit, une production. Aussi, l'arc narratif de la saison 4 ne fait que mettre à jour le dispositif fondamental de la série où du rien naît quelque chose. Plus que ce qui est produit, c'est bien le processus qui importe, le moment où ce qui n'est pas, ce qui semble infinitésimal, se transforme en quelque chose. D'un détail anodin surgit une interrogation dont la démesure fait le comique. Pour l'acteur, c'est le mouvement qui le conduit à endosser un rôle qui est mis en scène au gré de chaque épisode. On peut penser au jeu décalé de Jerry Seinfeld semblant parfois incarner à peine un rôle - ou peut-être n'est-il, comme le dit la productrice de NBC, qu'un comique de stand-up ne sachant pas jouer la comédie (4.24). George est évidemment la représentation extrême de cette capacité à endosser des rôles contradictoires et d'une impossibilité fondamentale à les conserver ${ }^{11}$. Logiquement, dans un temps où la sitcom lorgne vers le feuilleton en ménageant des évolutions aux personnages et en cherchant à utiliser le facteur temporel, Seinfeld sera marquée par une remarquable discontinuité : on laisse, au choix, George à la merci d'un tueur en série dans les toilettes d'un avion (4.12) ou dans les bras d'une amoureuse un peu trop passionnée (4.16) pour le retrouver seul et vivant, sans mémoire de ce passé embarrassant à l'épisode suivant. Chaque élément repris l'est dans une perspective non temporelle, sur le mode de la citation, du clin d'œil, dans un mouvement référentiel que nous avons déjà analysé.

Dans cette recherche du plaisir immédiat, qui naît d'un rien et se revendique infécond, c'est la culture du stand-up qui innerve la série (voir figure 9).

${ }^{11}$ Ce statut aléatoire du rôle et des signes qui le constituent est explicitement mis à jour par les auteurs dans l'épisode « The Opposite » (5. 24), où Elaine et George voient leur situation professionnelle évoluer de manière diamétralement opposée au même moment, tandis que Jerry voit sa situation changer pour devenir strictement identique à ce qu'elle était au départ. La part de l'expérimentation théorique l'emporte sur toute recherche de la vraisemblance. 


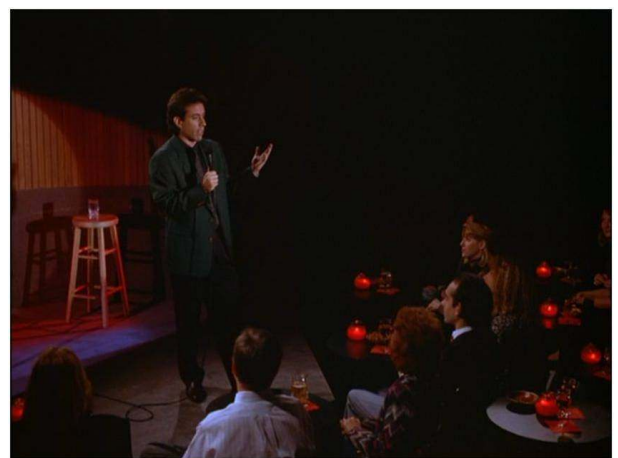

Fig. 9 : Le rire du stand-up : la culture de l'autoréférentiel

À travers un format qui reste parfaitement standardisé, cette culture fait exploser les codes de la sitcom. Mais ce dépassement est aussi celui du stand-up lui-même, dans la mesure où les auteurs créent d'épisode en épisode, de saison en saison, un écheveau complexe de signes, dans lequel importe plus la relation entre les éléments qu'une situation comique prise en elle-même.

Modestie et vanité se confondent dans le projet, comme le soulignent les auteurs dans le dialogue entre George et Jerry ${ }^{12}$. Le substantif « fun » résume à lui seul l'objectif visé par la création (voir figure 10). Débarrassée d'hypocrites fonctions qui alourdissent son contenu, la sitcom apparait pour ce qu'elle est : petit produit, fait de peu de choses dès lors que les conventions attachées aux personnages et aux intrigues sont abandonnées.

${ }_{12}$ À la fin de l'épisode « The Shoes » (4. 17), George et Jerry, contraints de s'enthousiasmer pour une idée d'Elaine, lui appliquent les termes qu'ils réservaient au dialogue qu'ils avaient eux-mêmes imaginé plus tôt dans l'épisode :

GEORGE. Well, it's a funny idea.

SEINFELD. It's funny.

GEORGE. Come on. Funny is funny.

SEINFELD. Funny's funny. We're here to entertain, right? 


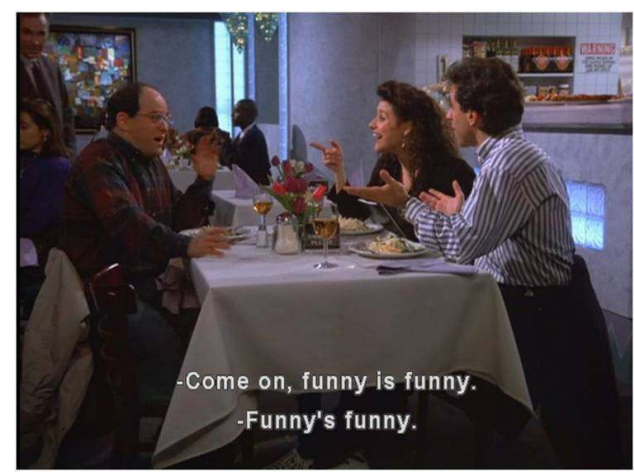

Fig. 10 : Faire rire : un projet dérisoire ou modeste ?

Les auteurs de Seinfeld veulent à chaque instant rappeler au téléspectateur le caractère purement artificiel de ce qui se joue à l'écran, mais aussi dans l'existence. Dans la série, on joue donc perpétuellement le jeu du dévoilement des mécanismes et d'exhibition des clichés. Si les personnages de Seinfeld sont à coup sûr des voyeurs, c'est bien pour que ceux qui les regardent prennent conscience du spectacle qu'on leur joue, des fictions improbables qu'on leur conte. Face aux voyeurs dupés, le téléspectateur retiendra enfin peut-être d'une création que le soupçon et la lucidité critique sont les deux seules attitudes pertinentes devant l'écran.

\section{Bibliographie}

Ahl Nils, FAu Benjamin (dir.), Dictionnaire des séries télévisées, Paris, Philippe Rey, 2011.

Colonna Vincent, L’Art des séries télé, Paris, Payot, 2010.

Gould Charlotte, "'Not That's There Anything Wrong With That': A Queer Reading of Seinfeld", GRAAT, issue \#2, June 2007, http://www.graat.fr/queertv04.pdf 


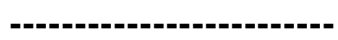

\section{L'auteur}

Éric Gatefin est docteur en Lettres Modernes (XVIII e siècle) et actuellement en poste en collège. Membre de l'équipe " Histoire des représentations » de l'université de Tours, il a publié plusieurs articles sur la littérature du XVIII siècle (notamment sur Diderot, le romanesque et les mémoires) et sa réception au XIX ${ }^{e}$ siècle. Auteur de contributions sur le cinéma fantastique de Jacques Tourneur et sur les films de Bruno Podalydès, il a également écrit deux articles sur The West Wing d'Aaron Sorkin ainsi qu'une contribution sur Homeland pour l'IFRI (Institut Français des Relations Internationales). 